\title{
Acoso escolar y consumo de drogas en estudiantes de educación media básica
}

\author{
Alberto Javier Córdova Alcaraz', Karina Jiménez Silvestre', Carmen Fernández Cáceres' \\ ' Centros de Integración Juvenil, A.C., México
}

\section{RESUMEN}

Introducción: el aumento del acoso escolar y de otras expresiones de violencia asociadas hace necesario estudiar su posible interacción con el uso de drogas, a fin de desarrollar alternativas preventivas adecuadas. Objetivo: contribuir a esclarecer la relación entre el acoso escolar y el consumo de drogas. Método: estudio transversal y comparativo con una muestra no probabilística de 1,025 estudiantes de educación secundaria, 55.3\% hombres y $44.7 \%$ mujeres, de entre 11 y 17 años. Se utilizaron el Auto-test de Cisneros y el Test de Agresión entre Pares para Adolescentes a fin de comparar, mediante la prueba de ANOVA, cuatro grupos (no usuarios de sustancias, usuarios de tabaco, de alcohol y de drogas ilegales); asimismo, se estimaron razones de momios mediante una regresión logística multinomial. Resultados: se observaron diferencias significativas entre grupos de usuarios y no usuarios, en términos de violencia, agresión física y amenazas; los usuarios de drogas ilícitas fueron quienes registraron calificaciones de violencia más altas. Se encontró también que ser víctima de intimidación y abuso reduce el riesgo de consumo, mientras que ser víctima de violencia física, ser propenso a reaccionar violentamente y presentar conductas desviantes lo aumenta. Discusión y conclusiones: a pesar de la evidencia de una asociación directa entre la violencia y el uso de sustancias, algunos de los hallazgos del estudio indican que ser víctima de acoso puede reducir el riesgo de consumo, por lo que es necesario realizar más estudios al respecto.

Palabras clave: adolescencia, acoso escolar, uso de drogas.

\begin{abstract}
Introduction: the increase in bullying practices and associated violence expressions requires the study of its possible interaction with drug abuse in order to develop appropriate prevention alternatives. Objective: to clarify the association between bullying and drug abuse. Method: a cross-sectional study was conducted, with a non-probabilistic sample of 1,025 junior high school students, 55.3\% men and $44.7 \%$ women, between 11 and 17 years old. Self-test of Cisneros and Peer Aggression Test for Teenagers were applied, comparing four groups by an ANOVA test (no substance users, tobacco users, alcohol users, and illegal drug users), and estimating odds ratios, by multinomial logistic regression. Results: significant differences between groups of users and non-users were detected, in terms of violence, physical aggression, and threatening; illicit drug users registered higher scores of violence. Being victim of some types of bullying, like intimidation and abuse, reduces the risk of drug abuse, meanwhile, being a victim of physical violence, violently reacting, and perform deviant behavior increase this risk. Discussion and conclusions: despite the evidence of a direct association between violence and drug abuse, some findings of this study indicate that being victim of bullying may reduce the risk of substance use, requiring further research.
\end{abstract}

Keywords: adolescence, bullying, drug use.

\section{Autor de correspondencia:}

Alberto Javier Córdova Alcaraz. Departamento de Investigación Psicosocial y Documental, Centros de Integración Juvenil, A.C. Tlaxcala núm. 208, piso 2, col. Hipódromo, del. Cuauhtémoc, C.P. 06100, Ciudad de México. Tel.:+52(55) 5999 4949, Ext. 7761. Correo electrónico: alberto.cordova@cij.gob.mx.

Recibido: 16 de marzo de 2016.

Aceptado: 18 de mayo de 2016.

DOI: 10.28931/riiad.2016.1.03 


\section{INTRODUCCIÓN}

El acoso escolar es un problema entre los adolescentes (Domínguez \& Manzo, 2011; Loredo-Abdalá, Perea-Martínez, \& López-Navarrete, 2008; Nansel et al., 2001; Olweus, 1993; Rettew \& Pawlowski, 2016) que se ha asociado, entre otros factores, con el consumo de drogas (Córdova, Ramón, Jiménez, \& Cruz, 2012; Perra, Fletcher, Bonell, Higgins, \& McCrystal, 2012); sin embargo, no resulta tan claro el sentido o la dirección en los que estos elementos se relacionan entre sí. En algunos casos se ha señalado que ser víctima de acoso predice el posterior uso de sustancias (Farrington \& Ttofi, 2011; Mitchell, Ybarra, \& Finkelhor, 2007), mientras que otros estudios afirman que utilizar drogas incrementa el riesgo de ser el perpetrador de la violencia (Jankauskiene, Kardelis, Sukys, \& Kardeliene, 2008; Romaní \& Gutiérrez, 2010). No obstante, la mayor parte de los estudios revisados -abocados a indagar la posible relación entre el acoso escolar y el consumo de sustancias- señala que existe una asociación entre el uso de alcohol, tabaco y drogas ilegales y el involucramiento en situaciones de acoso.

Los hallazgos parecen reforzar la hipótesis de que el consumo de alcohol es un predictor del acoso escolar, lo cual aplica tanto para el perpetrador de la violencia (Shamu et al., 2016; Siziya, Rudatsikira, \& Muula, 2012; Swahn et al., 2011; Topper, Castellanos-Ryan, Mackie, \& Conrod, 2011; Vaughn et al., 2010), como para la víctima (Fleming \& Jacobsen, 2009; King, Vidourek, \& Merianos, 2014; Kubwalo, Muula, Siziya, Pasupulati, \& Rudatsikira, 2013; Kuntsche \& Gmel, 2004; Moore et al., 2014; Radliff, Wheaton, Robinson, \& Morris, 2012; Siziya et al., 2012; Tharp-Taylor, Haviland, \& D’Amico, 2009).

Otros autores relacionan el uso de tabaco con el hecho de ser acosador (Azagba, 2016; Fleming \& Jacobsen, 2009; Jankauskiene et al., 2008; Topper et al. 2011), no obstante, algunos estudios concluyen que el uso de esta sustancia se vincula más con ser la víctima (Hazemba, Siziya, Muula, \& Rudatsikira, 2008; Kubwalo et al., 2013; Liang, Flisher, \& Lombard, 2007; TharpTaylor et al., 2009).

De la misma forma, se ha encontrado que el uso de drogas ilegales también predice la condición de víctima de acoso (Kelly et al., 2015; Radliff et al., 2012; Sullivan, Farrell, \& Kliewer, 2006; Tharp-Taylor et al., 2009; Vaughn et al., 2010).

Diversos estudios señalan una relación inversa, en la que las conductas de acoso pueden predecir el uso de sustancias (Elgar et al., 2014; Hawker \& Boulton, 2000; Lamont, Woodlief, \& Malone, 2014); según otras investigaciones, ser víctima de acoso también es un predictor del consumo (Radliff et al., 2012; Rudatsikira, Siziya,
Kazembe, \& Muula, 2007; Vieno, Gini, \& Santinello, 2011; Weiss, Mouttapa, Cen, Johnson, \& Unger, 2011).

Lamont y colaboradores (2014) afirmaron que el riesgo de fumar es menor entre los estudiantes acosados ya que se vuelven resilientes; esto contradice la afirmación de que el maltrato entre iguales es un factor que favorece las conductas de riesgo.

El presente estudio pretende identificar los elementos de exposición o ejercicio de la violencia que predicen el consumo de drogas, a fin de encontrar condiciones que den cuenta de la forma en la que los estudiantes se vinculan con el uso de sustancias.

\section{MÉTODO}

\section{Diseño}

Estudio transversal comparativo.

\section{Participantes}

A través de un muestreo no probabilístico se conformó una muestra de 1,025 estudiantes de nivel medio básico (secundaria), cuya distribución en los grupos cumplió, según cotejo en tablas, con un tamaño mínimo adecuado (46) para observar un efecto mediano de los factores (eta cuadrada $\left.\left[\eta^{2}\right]\right)$, con un alfa de $95 \%$ y $80 \%$ de poder estadístico (Jaccard \& Becker, 2002). Quedó integrada por estudiantes inscritos en escuelas previamente contactadas por personal de 10 unidades operativas ubicadas en Nuevo León, Tamaulipas y la Ciudad de México.

\section{Consumo de drogas}

Se conformaron cuatro grupos: el no consumidor, el consumidor de tabaco, el consumidor de alcohol y el consumidor de drogas ilegales, a partir del registro de uso "alguna vez en la vida" de tabaco, alcohol, mariguana, inhalables, cocaína, crack, estimulantes anfetamínicos, pastillas tranquilizantes, alucinógenos y heroína.

\section{Instrumentos}

Auto-test de Cisneros (Oñate, 2006; Piñuel \& Oñate, 2006), el cual explora tipos de victimización a través de un cuestionario de opción múltiple (1. Nunca, 2. Pocas veces y 3 . Muchas veces) que investiga los factores de violencia psicológica (nueve reactivos), violencia física (ocho reactivos), intimidación (cinco reactivos), desacreditación (seis reactivos) y abuso (tres reactivos), con una confiabilidad de .93 y una varianza explicada de $53.22 \%$. 
Test de Agresión entre pares para adolescentes (Cajigas de Segredo, Khan, Lizardo, Najson, \& Zalmavide, 2004), que explora diferentes tipos de ejercicio de la violencia a través de un cuestionario de opción múltiple (1. Totalmente en desacuerdo, 2. En desacuerdo, 3. Indeciso, 4. De acuerdo y 5. Totalmente de acuerdo) e identifica factores de agresividad (10 reactivos), conductas desviantes (cuatro reactivos) y violencia reactiva (cuatro reactivos), con una confiabilidad de .90 y una varianza explicada de $54.79 \%$.

Finalmente, la tercera cédula contiene una tabla para indagar consumo de sustancias alguna vez en la vida, en el último año y en el último mes.

\section{Procedimiento}

La aplicación de instrumentos se realizó en las escuelas secundarias seleccionadas, con previa autorización de los directores de cada uno de los planteles y la participación voluntaria de los jóvenes. La aplicación se realizó en las instalaciones escolares, de manera grupal, coordinada por el personal de Centros de Integración Juvenil.

\section{Análisis estadístico}

Se realizó un análisis descriptivo de los datos sociodemográficos, respecto a los indicadores de acoso escolar; se estimaron los índices promedio por factores y se compararon los puntajes entre los diferentes grupos de estudiantes conformados por medio de un análisis de varianza de una vía (ANOVA). Debido a que el objetivo principal fue identificar predictores del consumo, se realizó un análisis de regresión logística multinomial para obtener la razón de momios (OR, por sus siglas en inglés), tomando como referencia al grupo de no consumidores. Todos los procedimientos y análisis de la información se realizaron con el software SPSS V.20.

\section{RESULTADOS}

Integraron la muestra 567 hombres (55.3\%) y 458 mujeres $(44.7 \%)$, con un rango de edad entre 11 y 17 años, y una edad promedio de 13.59 ( $D E=1.08$ ); poco más de la mitad de los estudiantes (56.6\%) no reportó consumo de sustancias, $4.6 \%$ fumó tabaco, $23 \%$ consumió alcohol y $15.7 \%$ usó alguna droga ilegal.

Al comparar los cuatro grupos, en la escala de Cisneros (sobre exposición a la violencia), se encontraron diferencias significativas para el factor víctima de violencia física.

En la escala de Cajigas (sobre ejercicio de la violencia), se encontraron diferencias en los factores agresividad, conductas desviantes y violencia reactiva (Tabla 1).

En la Tabla 2 se exponen los resultados de las regresiones logísticas multinomiales, las cuales mostraron la relación de diversos factores de acoso escolar con el consumo de drogas.

Para el caso de los estudiantes que consumen alcohol, el hecho de ser víctima de intimidación y abuso reduce el riesgo de usar esta sustancia; por el contrario, vivir violencia física y ejercer violencia reactiva aumenta el riesgo de consumir alcohol.

En el grupo de los jóvenes que usan drogas ilegales, los estudiantes que han sufrido abuso por parte

Tabla 1

Diferencias en las áreas identificadas como acoso entre los no consumidores, consumidores de tabaco, consumidores de alcohol y consumidores de drogas ilegales

\begin{tabular}{|c|c|c|c|c|c|c|c|c|c|}
\hline & \multicolumn{2}{|c|}{$\begin{array}{c}\text { No } \\
\text { consumidores }\end{array}$} & \multicolumn{2}{|c|}{$\begin{array}{c}\text { Consumidores de } \\
\text { tabaco }\end{array}$} & \multicolumn{2}{|c|}{$\begin{array}{c}\text { Consumidores } \\
\text { de alcohol }\end{array}$} & \multicolumn{2}{|c|}{$\begin{array}{c}\text { Consumidores de } \\
\text { drogas ilegales }\end{array}$} & \multirow{2}{*}{$\begin{array}{c}\text { Anova } \\
F \\
g l=3\end{array}$} \\
\hline & Media & DE & Media & DE & Media & DE & Media & $\mathrm{DE}$ & \\
\hline \multicolumn{10}{|c|}{ Auto-test de Cisneros (víctima de la violencia) } \\
\hline Violencia psicológica & 1.26 & .378 & 1.29 & .262 & 1.30 & .357 & 1.28 & .353 & .798 \\
\hline Violencia física & 1.27 & .344 & 1.32 & .306 & 1.35 & .295 & 1.37 & .343 & $5.52^{*}$ \\
\hline Intimidación & 1.09 & .276 & 1.08 & .215 & 1.08 & .208 & 1.09 & .232 & .138 \\
\hline Desacreditación & 1.17 & .323 & 1.22 & .278 & 1.19 & .298 & 1.17 & .294 & .535 \\
\hline Abuso & 1.10 & .293 & 1.08 & .264 & 1.08 & .238 & 1.07 & .212 & .509 \\
\hline \multicolumn{10}{|c|}{ Test de Agresión de Cajigas de Segredo (ejercicio de la violencia) } \\
\hline Agresividad & 1.52 & 671 & 1.74 & .588 & 1.69 & 639 & 1.92 & .795 & $15.77^{\star}$ \\
\hline Conducta desviante & 1.48 & .626 & 1.63 & .591 & 1.67 & .646 & 1.92 & .809 & $19.53^{*}$ \\
\hline Violencia reactiva & 1.99 & .900 & 2.39 & .905 & 2.39 & .928 & 2.92 & 1.05 & $44.64^{*}$ \\
\hline
\end{tabular}


Tabla 2

Resultados de regresión logística multinomial de los factores de victimización y acoso escolar

\begin{tabular}{|c|c|c|c|c|c|c|c|c|c|}
\hline & \multicolumn{3}{|c|}{ Consumo de tabaco } & \multicolumn{3}{|c|}{ Consumo de alcohol } & \multicolumn{3}{|c|}{ Consumo de drogas ilegales } \\
\hline & \multirow{2}{*}{ OR } & \multicolumn{2}{|c|}{$\begin{array}{c}\text { Intervalo de } \\
\text { confianza }\end{array}$} & \multirow{2}{*}{ OR } & \multicolumn{2}{|c|}{$\begin{array}{c}\text { Intervalo de } \\
\text { confianza }\end{array}$} & \multirow{2}{*}{ OR } & \multicolumn{2}{|c|}{$\begin{array}{c}\text { Intervalo de con- } \\
\text { fianza }\end{array}$} \\
\hline & & $\begin{array}{l}\text { Límite } \\
\text { inferior }\end{array}$ & $\begin{array}{c}\text { Límite } \\
\text { superior }\end{array}$ & & $\begin{array}{l}\text { Límite } \\
\text { inferior }\end{array}$ & $\begin{array}{l}\text { Límite } \\
\text { superior }\end{array}$ & & $\begin{array}{l}\text { Límite } \\
\text { inferior }\end{array}$ & $\begin{array}{l}\text { Límite } \\
\text { superior }\end{array}$ \\
\hline \multicolumn{10}{|c|}{ Auto-test de Cisneros (víctima de la violencia) } \\
\hline Violencia psicológica & 1.04 & .269 & 1.02 & 1.26 & .626 & 2.57 & .987 & .411 & 2.37 \\
\hline Violencia física & 2.10 & .513 & 8.61 & $3.28^{* *}$ & 1.56 & 6.86 & $3.52^{\star \star}$ & 1.45 & 8.56 \\
\hline Intimidación & .360 & .060 & 2.16 & $.328^{\star}$ & .129 & .835 & .504 & .169 & 1.50 \\
\hline Desacreditación & 2.19 & .627 & 7.67 & 1.12 & .549 & 2.29 & .824 & .335 & 2.02 \\
\hline Abuso & .372 & .069 & 2.01 & $.400^{*}$ & .164 & .976 & $.313^{*}$ & .100 & .981 \\
\hline \multicolumn{10}{|c|}{ Test de Agresión de Cajigas de Segredo (ejercicio de la violencia) } \\
\hline Agresividad & 1.18 & .703 & 1.98 & .967 & .716 & 1.307 & .947 & .681 & 1.31 \\
\hline Conducta desviante & 1.06 & .621 & 1.81 & 1.24 & .934 & 1.66 & $1.55^{\star}$ & 1.12 & 2.13 \\
\hline Violencia reactiva & 1.41 & .972 & 2.06 & $1.44^{* *}$ & 1.184 & 1.76 & $2.40^{*}$ & 1.90 & 3.01 \\
\hline
\end{tabular}

Nota. La categoría de referencia es no consumo.

${ }^{\star} p<.05,{ }^{* *} p<.01$

de sus compañeros tienen menores posibilidades de consumir drogas, pero quienes han vivido violencia física son más propensos a usarlas. En este mismo grupo, quienes han ejercido sobre sus compañeros conductas desviantes o violencia reactiva presentan mayor riesgo de consumir.

En el grupo de uso de tabaco no se encontraron diferencias significativas, lo que indica que vincularse en actividades de acoso no predice el posterior consumo de tabaco, por lo menos en este grupo.

\section{DISCUSIÓN Y CONCLUSIONES}

Los resultados evidenciaron que los estudiantes con conductas de acoso escolar se encuentran con más frecuencia entre los consumidores de algún tipo de sustancia, tal como aseveran Jenson y Dieterich (2007). Asimismo, quienes presentan más conductas violentas son los usuarios de drogas -en comparación con los no usuarios-, quienes muestran conductas desviantes y de violencia reactiva.

De la misma forma, los que son víctimas de violencia, ya sea por intimidación o abuso, presentan un bajo riesgo de consumir drogas ilegales o alcohol. Lamont y colaboradores (2014) indicaron que la victimización puede fungir como un factor que reduce el riesgo de usar sustancias ilegales, por lo que sería conveniente realizar más estudios orientados a condiciones intervinientes como la resiliencia. No pasa lo mismo con los jóvenes que sufren violencia física, pues son más pro- pensos a usar alcohol o alguna droga ilegal (Farrington \& Ttofi, 2011; Mitchell et al., 2007).

Los usuarios de tabaco no parecen mostrar una vinculación significativa con las conductas de acoso, por lo menos en esta muestra. Pero los de alcohol sí presentan indicios de haber sido víctimas o perpetradores de violencia, como otros estudios señalan (Fleming \& Jacobsen, 2009; King et al., 2014; Kubwalo et al., 2013; Kunstche \& Gmel, 2004; Moore et al., 2014; Radliff et al., 2012; Sham, et al., 2016; Siziya et al., 2012; Swahn et al., 2011; Tharp-Taylor et al., 2009; Topper, 2011; Vaughn et al., 2010). Sería conveniente replicar el estudio en las zonas de influencia de los Centros de Integración Juvenil de todo el país, a fin de obtener una visión global de esta problemática, sus implicaciones y las intervenciones necesarias para aminorarla.

El reducido número de casos en el grupo de tabaco pudo ser un factor de influencia en los elementos predictores de este estudio, por lo que el resultado, en ese aspecto, se debe tomar con las reservas del caso.

Finalmente, el acoso escolar es una problemática que a nivel mundial se ha incrementado y propicia conductas de riesgo en los jóvenes. Cuando se asocia con el uso de sustancias, representa un problema de salud pública que demanda intervenciones tempranas e integrales que involucren de forma activa a los alumnos, las familias, el personal educativo y la sociedad en general, para la búsqueda de soluciones como la implementación de campañas de prevención del consumo de drogas en los diferentes ambientes donde se desenvuelven 
los jóvenes (Cerezo, Dolz, Pons-Salvador, \& Cantero, 1999; Mayer, 2008; Piñuel \& Oñate, 2006; Prieto, Carrillo, \& Jiménez, 2005; Tello, 2005).

\section{FINANCIAMIENTO}

Este estudio formó parte del Programa de Investigación de Centros de Integración Juvenil, A.C., por lo que no recibió financiamiento específico para su realización.

\section{CONFLICTO DE INTERÉS}

Los autores laboran en Centros de Integración Juvenil, A.C.; no obstante, el contenido del artículo representa su opinión y no necesariamente la de esta organización.

\section{REFERENCIAS}

Azagba, S. (2016). School bullying and susceptibility to smoking among never-tried cigarette smoking students. Preventive Medicine, 85(1), 69-73.

Cajigas de Segredo, N., Khan, E., Lizardo, M., Najson, S., \& Zalmavide, G. (2004). Escala de agresión entre pares para adolescentes y principales resultados. Acción Psicológica, 3(3), 173-186.

Cerezo, M. A., Dolz, L., Pons-Salvador, G., \& Cantero M. J. (1999). Prevención del maltrato de infantes: evaluación del impacto de un programa en el desarrollo de los niños. Anales de Psicología, 15(2), 239-250.

Córdova, A., Ramón, E., Jiménez, K., \& Cruz, C. (2012). Bullying y consumo de drogas (estudio documental). Revista de Psicología, Universidad de Antoquia, 4(2), 21-48.

Domínguez, L. F., \& Manzo, M. (2011). Las manifestaciones del bullying en adolescentes. Revista de Psicología, 8(17), 19-33.

Elgar, F. J., Napoletano, A., Saul, G., Dirks, M. A., Craig, W., Poteat, V. P., ... Koenig, B. W. (2014). Cyberbullying victimization and mental health in adolescents and the moderating role of family dinners. JAMA Pediatrics, 168(11), 1015-1022. doi:10.1001/jamapediatrics.2014.1223

Farrington, D. P., \& Ttofi, M. M. (2011). Bullying as a predictor of offending, violence and later life outcomes. Criminal Behaviour and Mental Health, 21(2), 90-98.

Fleming, L. C., \& Jacobsen, K. H. (2009). Bullying among middle-school students in low and middle income countries. Healt Promotion International, 25(1), 73-84. doi:10.1093/heapro/ dap046

Hawker, D., \& Boulton, M. (2000). Twenty years' research on peer victimization and psychosocial maladjustment: A meta-analytic review of cross-sectional studies. Journal of Child Psychology and Psychiatry, 41(04), 441-455.

Hazemba, A., Siziya, S., Muula, A. S., \& Rudatsikira, E. (2008). Prevalence and correlates of being bullied among in-school adolescents in Beijing: results from the 2003 Beijing Global School-Ba- sed Health Survey. Annals of General Psychiatry, 7(6), 1-6. doi:10.1186/1744-859X-7-6

Jaccard, J., \& Becker, M. (2002). Statistics for the behavioral sciences. [4ta. ed.]. Belmont, CA: Wadworth/Thompson Learning.

Jankauskiene, R., Kardelis, K., Sukys, S., \& Kardeliene, L. (2008). Associations between school bullying and psychosocial factors. Social Behavior and Personality: An International Journal, 36(2), 145-162.

Jenson, J., \& Dieterich, W. (2007). Effects of a skills-based prevention program on bullying and bully victimization among elementary school children. Prevention Science, 8(4), 285-296.

Kelly, E. V., Newton, N. C., Stapinski, L. A., Slade, T., Barrett, E. L., Conrod, P. J., ... Teesson, M. (2015). Concurrent and prospective associations between bullying victimization and substance use among Australian adolescents. Drug and Alcohol Dependence, 154(1),63-8. doi: 10.1016/j.drugalcdep.2015.06.012

King, K. A., Vidourek, R. A., \& Merianos, A. L. (2014). School violent victimization and recent alcohol use and episodic heavy drinking among youth. The Journal of School Nursing: The Official Publication of The National Association of School Nurses, 30(3), 187-195. doi:10.1177/1059840514521239

Kubwalo, H. W., Muula, A. S., Siziya, S., Pasupulati, S., \& Rudatsikira, E. (2013). Prevalence and correlates of being bullied among in-school adolescents in Malawi: results from the 2009 Global School-Based Health Survey. Malawi Medical Journal: The Journal of Medical Association of Malawi, 25(1), 12-14.

Kuntsche, E., \& Gmel, G. (2004). Emotional wellbeing and violence among social and solitary risky single occasion drinkers in adolescence. Addiction, 99(3), 331-339.

Lamont, A. E, Woodlief, D., \& Malone P. S. (2014). Predicting high-risk versus higher-risk substance use during late adolescence from early adolescent risk factors using Latent Class Analysis. Addiction Research and Theory, 22(1), 78-89.

Liang, H., Flisher, A. J., \& Lombard, C. J. (2007). Bullying, violence, and risk behavior in South African school students. Child Abuse \& Neglect, 31(2), 161-171.

Loredo-Abdalá, A., Perea-Martínez, A., \& López-Navarrete, G. E. (2008). "Bullying": acoso escolar. La violencia entre iguales. Problemática real en adolescentes. Acta Pediátrica Mexicana, 29(4), 210-214.

Mayer, L. (2008). Escuela, integración y conflicto. Notas para entender las tensiones en el aula. Educación Lenguaje y Sociedad, 6(6), 85-112.

Mitchell, K. J., Ybarra, M., \& Finkelhor, D. (2007). The relative importance of online victimization in understanding depression, delinquency and substance use. Child Maltreatment, 12(4), 314-324.

Moore, S. E., Norman, R. E., Sly, P. D. Whitehouse, A. J. O., Zubrick, S. R., \& Scott, J. (2014). Adolescent peer aggression and its association with mental health and substance use in an Australian cohort. Journal of Adolescence, 37(1),11-21.

Nansel, T. R., Overpeck, M., Pilla, R. S., Ruan, J., Simons-Morton, B., \& Scheidt, P. (2001). Bullying behaviors among US youth: prevalence and association with psychosocial adjustment. Journal of the American Medical Association, 285(16), 2094-2100. 
Olweus, D. (1993). Bullying at school. Oxford and Cambridge, MA: Blackwell Publishers.

Oñate, A. (2006). Acoso y violencia escolar. (Informe Cisneros X). Recuperado de: Instituto de Innovacion Educativa y Desarrollo Directivo: http://www.acosoescolar.com.

Perra, O., Fletcher, A., Bonell, C., Higgins, K., \& McCrystal, P. (2012). School-related predictors of smoking, drinking and drug use: Evidence from the Belfast Youth Development Study. Journal of Adolescence, 35(2), 315-324.

Piñuel, I., \& Oñate, A. (2006). Test AVE: acoso y violencia escolar. Madrid, España: TEA Ediciones.

Prieto, M., Carrillo, J., \& Jiménez, J. (2005). La violencia escolar; un estudio en el nivel medio superior. Revista Mexicana de Investigación Educativa, 10(27),1027-1045.

Radliff, K. M., Wheaton, J. E., Robinson, K., \& Morris, J. (2012). Illuminating the relationship between bullying and substance use among middle and high school youth. Addictive Behaviors, 37(4), 569-572.

Rettew, D. C., \& Pawlowski S. (2016). Bullying. Child and Adolescent Psychiatric Clinics of North America, 25(2), 235-242.

Romaní, F., \& Gutiérrez C. (2010). Auto-reporte de victimización escolar y factores asociados en escolares peruanos de educación secundaria, año 2007. Revista Peruana de Epidemiología, 14(3), 1-9.

Rudatsikira, E., Siziya, S., Kazembe, L. N., \& Muula, A. S. (2007). Prevalence and associated factors of physical fighting among school-going adolescents in Namibia. Annals of General Psychiatry, 6(7), 1-5.

Shamu, S., Gevers, A., Mahlangu, B. P., Jama Shai, P. N., Chirwa, E. D., \& Jewkes, R. K. (2016). Prevalence and risk factors for intimate partner violence among grade 8 learners in urban South Africa: baseline analysis from the Skhokho Supporting Success cluster randomised controlled trial. International Health, 8(1), 1826. doi:10.1093/inthealth/ihv068
Siziya, S., Rudatsikira, E., \& Muula, A. S. (2012). Victimization from bullying among school-attending adolescents in grades 7 to 10 in Zambia. Journal of Injury \& Violence Research, 4(1), 30-35. doi:10.5249/jivr.v4i1.84

Sullivan, T., Farrell, A., \& Kliewer, W. (2006). Peer victimization in early adolescence: Association between physical and relational victimization and drug use, aggression, and delinquent behaviors among urban middle school students. Developmental Psychopathology, 18(1), 119-137.

Swahn, M. H., Topalli, V., Ali,B., Strasser, S. M., Ashby, J. S., \& Meyers, J. (2011). Pre-teen alcohol use as a risk factor for victimization and perpetration of bullying among middle and high school students in Georgia. The Western Journal Of Emergency Medicine, 12(3), 305-309.

Tello, N., (2005). La socialización de la violencia en las escuelas secundarias. Proceso funcional a la descomposición social. Revista Mexicana de Investigación Educativa, 10(27),1165-1181.

Tharp-Taylor, S., Haviland, A., \& D'Amico, E. J. (2009). Victimization from mental and physical bullying and substance use in early adolescence. Addictive Behaviors, 34(6), 561-567.

Topper, L. R., Castellanos-Ryan, N., Mackie, C., \& Conrod, P. J. (2011). Adolescent bullying victimisation and alcohol-related problem behaviour mediated by coping drinking motives over a 12 month period. Addictive Behaviors, 36(1-2), 6-13.

Vaughn, M. G., Qiang, F., Bender, K., DeLisi, M., Beaver, K. M., Perron, B. E., ... Howard, M.O. (2010). Psychiatric correlates of bullying in the United States: findings from a national sample. Psychiatric Quarterly, 81(3), 183-195.

Vieno, A., Gini, G., \& Santinello, M. (2011). Different forms of bullying and their association to smoking and drinking behavior in italian adolescents. Journal of School Health, 81(7), 393-399.

Weiss, J. W., Mouttapa, M., Cen, S., Johnson, C., \& Unger, J. (2011). Longitudinal effects of hostility, depression, and bullying on adolescent smoking initiation. Journal of Adolescent Health, 48(6), 591-596. 